\title{
Revisión sobre los efectos de estudiar con música de fondo y sobre los efectos del entrenamiento musical en el aprendizaje
}

\section{Available research on the use of background music during studying, and the ef- fects of music training in learning}

\author{
Cantú Cervantes, Daniel ${ }^{1}$
}

Abstract:

\begin{abstract}
Resumen:
Se analizaron dos aspectos importantes de la música en el aprendizaje, por un lado, los efectos de la música de fondo durante el estudio académico en los estudiantes, y por otra parte, los efectos del entrenamiento musical en el aprendizaje. Se realizó una revisión bibliográfica en las bases de datos ScienceDirect, Scopus, PubMed y Google Scholar sobre las palabras clave: música de fondo, música aprendizaje, música estudio y entrenamiento musical; incluyendo artículos, capítulos y libros especializados en inglés y español publicados desde 1998 hasta mayo de 2020, con el propósito de exponer un panorama actualizado sobre los hallazgos y efectos del impacto de la presencia de música en el estudio académico y sobre las ventajas que brinda el entrenamiento musical respecto al aprendizaje. La evidencia sugiere que música de fondo perturba más la concentración en el estudio académico que la condición de silencio. Por otro lado, se identificó que los efectos benéficos del entrenamiento musical son consistentes con cuatro áreas académicas: el lenguaje, cálculo mental, representación de artes escénicas y la orientación espacial manual.
\end{abstract}

Palabras Clave: música de fondo; estudio académico; aprendizaje; estudiantes; entrenamiento musical.

${ }^{1}$ Universidad Autónoma de Tamaulipas

*Correspondencia: dcantu@docentes.uat.edu.mx
This study aimed to analyze two essential aspects of music in learning. On the one hand, the effects of the use of background music during academic studying. And, on the other hand, the effects of music training in learning. We search in four databases (1998 to May 2020) to identify relevant articles, chapters, and specialized books related to background music in the academic study and the music training in learning. All this shows an updated panorama on the effects of background music during academic studying and, benefits of training music in the learning. The evidence suggests that background music disturbs more the concentration in the academic study that the silence condition. On the other hand, the effects of music training are consistent with the benefit of four academic areas: language, mental calculation, performing arts and, manual spatial orientation.

Keywords:background music; academic study; learning; music training. 
La música puede considerarse como un estímulo psicobiológico sensorial especial, cognitivo emocional complejo y motor, de sonidos organizados en el tiempo que promueven imaginerías mentales inefables y episódicas que se generan de acuerdo con la experiencia de cada sujeto (Talamini, Grassi, Toffalini, Santoni \& Carretti, 2018; Ben-Nathan, Salti \& Algom, 2019). La complejidad de la música radica en la estructuración de la articulación y la combinación de sonidos en el espacio del tiempo con la superposición de ritmo, armonía y melodía. El ritmo es la sucesión de sonidos situada bajo patrones de fraseos articulados en intervalos de duración (Carrasco, Gustems y Calderón, 2016); la melodía es la combinación del ritmo bajo alturas tonales que estructuran frases musicales (Kim, Lee, Kim, Seol, Yi \& Chung, 2014), y la armonía es la asociación de dichos sonidos y frases que de forma simultánea son superpuestos en sucesiones ordenadas (Trost, Labbé \& Grandjean 2017).

La comprensión musical requiere del análisis de patrones acústicos constituidos por la intensidad y la frecuencia de los inicios y las duraciones tonales de la melodía, y de las frases armoniosas (Matute, 2012), de manera que como explican Khan, Kitsis, Golovyan, Wang, Chlan, Boustani \& Khan (2018), el sujeto le confiere a partir de su experiencia, una identidad y significado emocional a cada pieza musical. En este respecto, a través de una historia de aprendizaje musical, estas asociaciones permiten que la música pueda agruparse por géneros, al tiempo que genera en cada sujeto, gustos y preferencias musicales. Flanagan \& Alfonso (2016) y Christopher \& Shelton (2017), señalan que escuchar una pieza musical requiere de atención, percepción, decodificación y por supuesto del bagaje cognoscitivo que le permita al sujeto, identificar y retener las relaciones espaciales y secuenciales de la estructuración de la pieza musical para generar un sentido sobre las expectativas de la consecución de los sonidos en el tiempo. Cantú, Lera y Baca (2017) y Bashwiner \& Bacon (2019), indican que, si bien la activación del córtex en la escucha musical es múltiple, existe una activación preponderante de las zonas temporales y emocionales del sistema límbico, además de la participación cortical asociativa de ambos hemisferios.

Wiley \& Jarosz (2012) y Mitchell (2018), señalan que las implicaciones cognitivas de la atención y percepción durante la escucha de la música requieren de la intervención directa de la memoria de trabajo, que es crítica durante situaciones de concentración como lo es el estudio autónomo. Sweller (2003), Baddeley (2007) y Flanagan \& Alfonso (2016), afirman que la implicación de la memoria de trabajo en la escucha de la música incide en la retención de los componentes sonoros y sus relaciones, lo que genera una carga para el procesamiento de la atención sostenida. En este sentido, la memoria de trabajo genera mediante el razonamiento redes temporales diferenciadas con alto grado de inhibición para discriminar informaciones no relevantes y centrar la atención en el contenido musical percibido para su proceso y almacenaje en redes engrama de la memoria a largo plazo; sin embargo, como señalan Engle \& Kane (2003) y Burunat, Alluri, Toiviainen, Numminen \& Brattico (2014), este tipo de memoria que conforma la base para la codificación mnémica, es muy susceptible a la interferencia y además posee una capacidad limitada que se desvanece pronto, a menos que se refresque mediante el repaso.

Cuando la música es preferida para el sujeto, puede mejorar su motivación en tareas motoras repetitivas, o que requieren del es- 
fuerzo físico como el deporte; incluso en tareas motoras finas como el ejercicio quirúrgico (Boghdady \& Ewalds-Kvist, 2020), sin embargo, si bien estos beneficios suelen darse durante tareas manuales, Penagos-Corzo y De La Fuente (2012) y Custodio y Cano (2017), indican que no acontece así en tareas de concentración no manual como el estudio académico, ya que como explica Li, Chen \& Tsai (2019), entre más preferida sea la pieza musical para la persona, se genera un mayor incremento emocional que desencadena una serie de imaginerías mentales y recuerdos con alto contenido emocional, que pueden facilitar un sesgo en la desconcentración durante tareas de estudio académico.

¿Puede la música favorecer de alguna manera el aprendizaje?, más allá de la música de fondo en el estudio académico, se encuentra el entrenamiento musical que promueve (más que el mero hecho de escuchar música) la producción creativa auditiva, emocional y motora articulada de piezas musicales (Hardin, Sammler, Henry, Large \& Kotz, 2019). Chiang, Rosenberg, Bufford, Stephens, Lysy \& Monti (2018) señalan que la producción musical circunda en una ejecución mental que requiere de la generación de ajustes tonales, discriminación melódica y una elaboración creativa de largas secuencias sonoras y de reconocimiento espacial sobre el espectro de patrones acústicos modulados en el tiempo; todo esto como indican Jicol, Proulx, Pollick \& Pretini (2018), mediante la modulación de niveles de intensidad y frecuencia respecto a las duraciones tonales de una melodía armoniosa. En este sentido, Graziano, Peterson \& Shaw (2016) y Sala \& Gobet (2017), señalan que el entrenamiento musical adecuado genera una coordinación motora y espacial fina más desarrollada, debido al incremento de la sensibilidad tonal para generar por medio de la acción motora, mo- dulaciones de frecuencia y presión sobre los acordes para producir cadencias melódicas. McKetton, DeSimone \& Schneider (2019) identificaron que el entrenamiento musical adecuado mostró incrementos morfológicos de sustancia gris en las regiones primaria y rostral temporal de la corteza auditiva, y en el grosor de sustancia blanca del cuerpo calloso. Por su parte, Angulo-Perkins, Aubé, Peretz, Barrios, Armony \& Concha (2014), reportaron que el planum polare (parte del giro temporal superior en el lóbulo temporal) en los músicos, muestra una actividad preferencial en respuesta a estímulos musicales, independientemente de la tipología de instrumento ejecutado. Por otro lado, Habibi, Damasio, Ilari, Joshi, Leahy, Haldar, ... \& Damasio, H. (2018), identificaron un aumento de sustancia blanca en el cuerpo calloso de personas con entrenamiento musical, específicamente en las vías de cruce que conectan segmentos frontales, sensoriales y motores superiores (datos similares reportados por Butera, 2015; Allen, Burton, Olman \& Oxenham (2017). En este respecto, Gaser \& Schlaug (2003) y Manes y Niro (2014), señalan que el entrenamiento musical favorece la sensibilidad emocional que impacta en una mayor asociación mnémica, ya que el incremento emocional fortalece el traspaso proteico sináptico de las redes engrama que albergan los recuerdos, beneficiando su reminiscencia.

Ante estas implicaciones, Doyle \& Furnham (2012), Rosen, Carrier \& Cheever (2013) y Bellur, Nowak \& Hull (2015), afirman que escuchar música mientras se estudia se ha convertido en una práctica habitual entre los alumnos universitarios y de nivel medio superior, que además tienden a estudiar con otros distractores, como el uso simultáneo de las redes sociales y dispositivos móviles, todo esto como explican Ransdell \& Gilroy (2001); Dobbs, Furnham \& McClelland 
(2011) y Calderwood, Ackerman \& Conklin (2014) a pesar de estar conscientes de que la eficacia para la concentración puede resultar afectada. En este respecto, Hallyday (2019), señala que cuanto más el sujeto está expuesto a la música desde temprana edad, es más propenso a reunir herramientas que le permitan involucrar la música en todas sus actividades, incluyendo el estudio académico. Judd (2014) y Xu, Wang \& David (2016), indican que un buen número de estudiantes considera que la música puede ayudarlos a combatir la somnolencia durante el estudio, e incluso favorecer su aprendizaje autónomo; sin embargo, como indican Lehmann \& Seufert (2017), escuchar música durante tareas de estudio académico representa un obstáculo para la atención que puede dificultar la concentración.

Desde luego la música con letra puede usarse y tener resultados benéficos cuando el mensaje se relaciona directamente con el contenido a aprender, sin embargo, cuando la pieza musical prosódica no tiene relación con la tarea de estudio, se convierte en un factor distractor (Widerman, 2013). Es en este sentido, y dadas las implicaciones precedentes, que surge la pregunta de investigación: ¿cuáles son los efectos de estudiar con presencia de música de fondo, y cuáles son los efectos más sobresalientes del entrenamiento musical en el aprendizaje?

\section{Metodología}

Se llevó a cabo una revisión bibliográfica manual en las bases de datos ScienceDirect, Scopus, PubMed y Google Scholar sobre las palabras clave "música de fondo", "música aprendizaje", "música estudio" y "entrenamiento musical" en trabajos publicados desde 1998 hasta mayo de 2020. La búsqueda se realizó sin restricción en los campos, y se procuró una búsqueda bibliográfica itera- tiva, para encontrar materiales hasta alcanzar una saturación teórica. Se dio prioridad a los estudios más recientes, y se usó la combinación de sinónimos y términos relacionados con las palabras clave de la búsqueda; además se incluyeron las conjunciones "and" y "or". En este sentido, solo se incluyeron artículos científicos, capítulos de libro publicados y libros especializados, descartando ponencias de congresos, cartas e introducciones editoriales de revistas. La búsqueda se extendió hacia la aceptación de trabajos en cualquier idioma incluyendo los artículos relacionados con los temas que se encontraban en las referencias de los previamente incluidos. Por otra parte, se excluyeron aquellos trabajos publicados que no se relacionaban directamente con los efectos de la música y del entrenamiento musical en el aprendizaje. El filtro de selección se realizó en primer lugar por título del artículo o capítulo, luego por el abstract o resumen, y finalmente por el contenido del trabajo. Se estableció la siguiente hipótesis de trabajo: el uso de la música de fondo es más disruptiva para la concentración que la condición de silencio durante el estudio autónomo académico; además, el entrenamiento musical adecuado produce mayores ventajas cognitivas que el hecho de escuchar música antes o durante el estudio académico.

\section{Limitaciones de investigación}

Las limitantes de esta revisión giraron en virtud de no describir con profundidad las zonas neurofisiológicas que se activan o participan durante la escucha de la música en el estudio académico, o durante el entrenamiento musical. En este sentido, no se enfatizó en comprender con profundidad las relaciones y las diferencias entre las zonas neurales preponderantes y participantes durante la presencia de la música en el estudio académico y en el en- 
trenamiento musical. Por otro lado, no se procuró profundizar en las diferencias de los efectos de la música de fondo en el estudio académico entre músicos y no músicos, ni se ahondó en los efectos del canto en el aprendizaje, ni en la etiológica cognitiva y congénita de los sujetos con sentido musical nato y de los músicos no natos que reciben entrenamiento musical. Solo se enfatizó en conocer los efectos dados cuando los alumnos en general estudian con presencia de música de fondo, y cuando lo hacen en otras circunstancias como durante la condición de silencio; además, se priorizó en rescatar los efectos más sobresalientes del entrenamiento musical sobre el estudio y el aprendizaje.

En este sentido, se presenta a continuación un primer apartado que muestra una serie de estudios que han reportado efectos sobre la música de fondo en el estudio académico, y un segundo apartado más adelante que muestra los efectos más sobresalientes del entrenamiento musical en el estudio y el aprendizaje. Seguidamente se presentan las conclusiones con los hallazgos más relevantes, y el corpus de las referencias bibliográficas de trabajo.

\section{Hallazgos sobre los efectos de la música de fondo en el estudio académico y el aprendi- zaje}

Es necesario indicar previa y brevemente aquellos contextos no académicos donde la música de fondo ha mostrado bondades favorecedoras; por ejemplo, la música de fondo muestra por lo general un impacto positivo en el incremento de las ventas en comercios departamentales (Kang \& Lakshmanan, 2017; Hsing-Chi \& Oh, 2020; Wen, Leung \& Pongtornphurt, 2020), aunque también se ha identificado que los consumidores muestran disgusto y molestia cuando las canciones no son de su agrado, disminuyendo el consumo de dichos compradores (Hynes \& Manson, 2016;
Michel, Braumann \& Gayer, 2017). Por otro lado, la música favorece ambientes relajantes que combaten la ansiedad en las salas de espera de los hospitales (Gutgsell, Schluchter, Margevicius, DeGolia, McLaughlin, Harris, ... \& Wiencek, 2013), favorece la sedación (Kipnis, Tabak \& Koton, 2016), y ha mostrado ventajas durante tratamientos preoperatorios y cirugías, ya que las piezas musicales facilitan la relajación y modifican las respuestas vasculares para tener un mejor control quirúrgico (Ni, Tsai, Lee, Kao \& Chen, 2012; McClurkin \& Smith, 2016), aunque también se ha identificado que estos beneficios aumentan cuando la música es preferida y seleccionada por parte de los pacientes (Akelma, Altinsoy, Arslan \& Ergil, 2020).

Por otro lado, la música ameniza y alarga la estadía en las reuniones casuales (Daykin, Mansfield, Meads, Julier, Tomlinson, Payne, ... \& Victor, 2018), y se ha convertido en un aliado de las terapias conductuales y lingüísticas para tratar trastornos como la afasia no afluente (Geretsegger, Elefant, Mössler \& Gold, 2014; Galińska, 2015; Biasuti, 2019). Además, Shang \& Gao (2014) y Aljanaki, Wiering \& Veltkamp (2016), indican que la música a alto volumen con ritmo rápido genera un efecto motivacional en el rendimiento físico en gimnasios y entrenamientos de alto rendimiento, no obstante, Chiat \& Fung (2012), señalan que es recomendable que los entrenadores conozcan los antecedentes de los gustos musicales de los deportistas para mejorar las rutinas. Por otro lado, se ha identificado que la música puede relajar a los bebés recién nacidos y mejorar sus ciclos de lactancia y sueño (JohansenBerg, 2001; Ettenberger \& Beltrán, 2018; Robertson \& Detmer, 2019), sin embargo, Damasio (2006) y Guerra \& Muñoz de Rodríguez (2013), indican que más allá de la música o de la buena entonación de la madre, los bebés se tranquilizan con el habla y canto de 
la madre, aun cuando éste no sea rítmico, melódico o muy elaborado.

En el contexto académico del estudio autónomo, Landay \& Harms (2018), señalan que la música favorece el estado de ánimo y la motivación, siempre y cuando las piezas musicales sean preferidas por el usuario; en este sentido, Groot (2006), Perham \& Vizard (2011) y Penagos-Corzo y De La Fuente (2012), indicaron que cuanto más preferida es la música, mayor es el incremento emocional que provoca una mayor cantidad de imaginerías mentales y recuerdos emocionales que interrumpen la concentración en el estudio autónomo. Estos hallazgos concuerdan con los reportados por Perham \& Sykora (2012) y Li et al. (2019), que señalaron que la música preferida provocó un menor rendimiento en el estudio académico de los estudiantes, que la música desconocida o no preferida; reportando también un mayor incremento emocional cuando los alumnos escucharon la música que les gustaba. Por su parte, Furnham \& Allas (1999) y Jäncke \& Sandmann (2010), encontraron puntajes más bajos en los alumnos que estudiaron con música conocida que con música desconocida, identificando además que la música a ritmo rápido perturbó con mayor frecuencia a los estudiantes durante el estudio académico que la música a ritmo lento. Datos similares también reportados por Hallam, Price \& Katsarou (2010).

Mohan \& Thomas (2020) y Hallyday (2019), indican que la música de fondo se encuentra presente con mayor frecuencia en el estudio académico, cuando el estudiante fue propenso a escuchar música a menudo desde edad temprana. En este sentido, Črnčec, Wilson \& Prior (2006) y Mowsesian \& Heyer (2018) señalan que la mayoría de los alumnos en la actualidad, pueden tratar a la música de fondo como un sonido irrelevante dada la ex- cesiva estimulación digital y simultánea entre el uso de redes sociales, videojuegos, televisión y dispositivos móviles usados durante el estudio académico, sin embargo, Johansson, Holmqvist, Mossberg \& Lindgren (2012) y Zhang, Miller, Cleveland \& Cortina (2018), indican a pesar de que los estudiantes intenten ignorar o tratar a la música de fondo como un sonido irrelevante durante el estudio académico, la música bifurca la atención haciéndola más intermitente que simultánea, es decir, que se generan rápidos $\mathrm{y}$ constantes momentos volátiles donde se coloca atención al estudio, con momentos muy rápidos de atención consciente hacia la pieza musical, haciendo más lento el proceso de estudio académico.

Por su parte, Salamé \& Baddeley (2007) identificaron que la memoria de trabajo se interrumpe con frecuencia cuando se estudia con música de fondo instrumental, y en mayor frecuencia cuando dicha música contiene lírica, incluso si el estudiante hace el esfuerzo por no prestarle atención o cuando se trata de una pieza musical en un idioma extranjero no entendible para el estudiante (por ejemplo, el inglés para alumnos hispanohablantes). Estos investigadores (Salamé \& Baddeley, 2007), también identificaron que el silencio resultó menos perturbador que cualquier estímulo sonoro durante las tareas de estudio en clase (resultados similares también reportados por Kang \& Lakshmanan, 2017; Schiller, Morsomme \& Remacle, 2018; Echaide, Del Río \& Pacios 2019; Koelsch, Vuust \& Fritson, 2019). Así mismo, Tervaniemi, Szameitat, Kruck, Schröger, Alter, De Baene \& Friederici (2006), indicaron que la música instrumental fue menos perturbadora en el estudio académico que las canciones con lírica, sugiriendo que la semántica de las letras al tener un fin comunicativo, terminan por atraer más la atención del escucha, ya que 
la mayoría de las canciones contienen mensajes e historias con tinte sentimental que impiden que el oyente trate de ignorarlas por completo.

Por otro lado, Kumar, Wajidi, Chian, Vishroothi, Ravindra \& Aithal (2016), indicaron que cuando los alumnos estudiaron con música de fondo instrumental con ritmo lento, obtuvieron mejores puntajes que cuando el ritmo era rápido (datos similares reportados por Furnham \& Allas, 1999, y Jäncke \& Sandmann, 2010), mientras que Perham \& Currie (2014) identificaron que la música con prosodia perturbó la concentración lectora durante el estudio autónomo, aun cuando las piezas musicales fueron preferidas por los estudiantes. En este respecto, Perham \& Vizard (2011), ya habían informado que las piezas musicales preferidas o no, mostraban una mayor interrupción que la condición de silencio durante tareas de concentración en el estudio académico. Por otro lado, Furnham \& Strbac (2010) encontraron que tanto la música como el ruido a bajo volumen (sonido de personas trabajando o conversando) generan una mayor interrupción en la concentración durante el estudio académico que la situación de silencio. En este sentido, Kaarlela-Tuomaala, Helenius, Keskinen \& Hongisto (2009) y Banbury \& Berry (2010), afirman que por lo general, los esfuerzos de las personas por ignorar el ruido no tienen éxito cuando la escuela o las oficinas se encuentran cerca de centros de trabajo que provocan mucho escándalo sonoro.

Respecto a las tareas académicas manuales y no manuales, Penagos-Corzo y De La Fuente (2012), informaron que la música de fondo puede favorecer la realización de tareas académicas manuales, pero entorpece la concentración en tareas de estudio académico (no manuales). Sin embargo, Davidson
\& Powell (2015), identificaron que la música de fondo mejoró el desempeño de tareas académicas no manuales cuando se combinaron con tareas manuales, por ejemplo, en actividades de laboratorio químico escolar, donde el alumno realiza acciones manuales al tiempo que se revisa la literatura. Por otra parte, Miché (2002) y Salmon (2009), identificaron que la música combinada con el canto y la acción manual de los ademanes, favorece más los aprendizajes que el mero hecho de escuchar las piezas musicales. En este sentido, Hafteck (2006) y Feierabend (2010), también indicaron que el canto con ademanes en clase (aunque puede no formar parte del entrenamiento musical adecuado) siempre que tenga que ver con los contenidos a aprender, propicia una mejor aprehensión del vocabulario natal o de otro idioma extranjero, incluso puede favorecer la alfabetización y las habilidades del habla en niños de preescolar.

Los estudios que se muestran a continuación, presentan hallazgos sobre el impacto de la música de fondo en diversas actividades de estudio y pruebas académicas, sin embargo, cabe indicar, que estos hallazgos pueden parecer no consistentes ya que algunos encontraron que la música favoreció el puntaje en determinadas actividades académicas con música de fondo y otros indicaron mayor perturbación durante la escucha de la música; además, no todos los estudios se centraron exactamente en las mismas habilidades específicas, por ejemplo, Lamb \& Gregory (2006) y Bloor (2009) trabajaron con estudiantes de educación elemental mediante pruebas de conocimiento general en presencia de música de fondo y encontraron puntajes más altos en lectura, pero bajos puntajes y mayor distracción en tareas sobre matemáticas; aunque por otro lado, Taylor \& Rowe (2014), reportaron mejores puntajes en matemáticas en alumnos 
universitarios cuando realizaron pruebas con música de fondo. Por otra parte, Ransdell \& Gilroy (2001), identificaron una mayor perturbación con bajos puntajes en tareas de escritura en presencia de música de fondo durante las actividades de estudio en clase.

Por su parte, Davidson \& Powell (2015) reportaron mejores puntajes en los alumnos (sin distinción de género) que estudiaron con presencia de música de fondo, aunque en esta investigación no se contó con un grupo de comparación. En este sentido, bajo un contexto de comparación entre grupo experimental y de control, Lehmann \& Seufert (2017) no identificaron diferencias importantes entre los puntajes de los alumnos que estudiaron con música de fondo que aquellos que estudiaron en silencio, mientras que Dosseville, Laborde \& Scelles (2012), indicaron que los alumnos que estudiaron por medio de videos instruccionales con música de fondo, presentaron mejores puntajes que los del grupo de control que estudiaron con los mismos videos sin música (otros estudios como Lozano y Lozano, 2007; Neville, Andersson, Bagdade, Bell, Currin, Fanning., ... \& Yamada, 2008; Charif, Mitrofan \& Gâtej, 2014; Simhon, Elefant \& Orkibi, 2018, también reportaron resultados similares a los encontrados por Dosseville et al., 2012, y Lehmann \& Seufert, 2017). Bajo estas implicaciones y por su parte, Jeong \& Ryu (2016), observaron mejores puntajes en alumnos durante actividades de estudio en clase con presencia de música de fondo, pero solo cuando se trabajó con materiales que contenían información no verbal.

Existe una mayor cantidad de estudios que reportan bajos puntajes en el estudio académico en presencia de música de fondo, por ejemplo, Lehmann, Hamm \& Seufert (2018), indicaron que los estudiantes pudieron recordar más información cuando estudiaron en silencio, que cuando estudiaron con música de fondo. Al respecto, Salamé \& Baddeley (2007) y Furnham \& Strbac (2010) observaron que tanto el ruido de la televisión como la música de fondo, resultaron más disruptivos en el estudio académico que la condición de silencio (datos similares también identificados por Moreno \& Mayer, 2010; Kang \& Lakshmanan, 2017; Schiller et al., 2018). Por otra parte, Perham \& Vizard (2011), reportaron un mayor puntaje durante el estudio autónomo en silencio que cuando los alumnos estudiaron en presencia de música, e incluso cuando las piezas musicales fueron preferidas o no por los estudiantes.

Reynolds, McClelland \& Furnham (2014) por otro lado, identificaron que la música de fondo perturbó más a los estudiantes durante tareas de cálculo mental que la condición de silencio. Perham \& Sykora (2012), informaron que los alumnos que estudiaron con música preferida obtuvieron puntajes más bajos que cuando estudiaron con música no preferida, observando los puntajes más altos cuando estudiaron en silencio. Asimismo, Dobbs et al. (2011), indicaron que la música de fondo como el ruido blanco o rosa perturbaron más a los estudiantes durante el estudio académico que la condición de silencio. Otros estudios como el de Zhang et al. (2018), informaron que los alumnos que estudian con música de fondo, tienden a desviar la mirada de la lectura o del material de estudio con más frecuencia que aquellos que estudian en un ambiente de silencio. En este respecto, Johansson et al. (2012), señalan que la desatención del estudio en presencia de música de fondo a pesar de ser frecuente, es muy sutil que puede generar que el alumno no se percate de ello. Estos autores (Johansson et al., 2012), al igual que Anderson \& Fuller (2010) y Cauchard, Cane \& Weger (2012), encontra- 
ron bajos puntajes en el estudio autónomo en presencia de música de fondo que cuando los alumnos estudiaron en silencio. Resultados similares fueron reportados por Oswald, Tremblay \& Jones (2000), Anderson, Henke, McLaughlin, Ripp \& Tuffs (2000), Pool, Koolstra \& Van Der Voort (2003), Neville et al. (2008), Christopher \& Shelton (2017) y Khan et al. (2018).

Sobre las implicaciones del silencio en la atención y en el estudio académico, Olsen (2013) y Bigliassi, Karageorghis, Bishop, Nowicky \& Wright (2018), indican que tanto en la radio como en la televisión, se acostumbra a prescindir de la música de fondo durante el voceo de mensajes, noticias y avisos muy trascendentes con el fin de que el oyente aprecie los detalles más específicos del mensaje. Un ejemplo clásico de la comprensión del silencio en el estudio académico como señala Khan et al. (2018), son las bibliotecas, que de forma general y contemporánea han ilustrado el concepto de la perturbación sonora durante la concentración en el estudio autónomo.

Por último, cabe realizar algunas aproximaciones hacia el efecto Mozart, que se refería a la propuesta de una sugestiva mejora de las habilidades temporo-espaciales después de escuchar una sonata de piano del compositor Wolfgang Amadeus Mozart (Rauscher \& Shaw, 1998; Rausher \& Hinton, 2010). La creencia en el efecto Mozart se dilató y llegó a ser tal, que algunos hospitales regalaban discos compactos con música de Mozart a las madres embarazadas para que el bebé naciera "más inteligente" que los demás de su generación, dada la mera melódica cadencia de las sonatas (Talero-Gutiérrez y Saade-Lemus, 2018). Incluso se llegó a suponer que el efecto Mozart podía curar enfermedades y favorecer la creatividad con el simple hecho de escuchar las piezas musicales del compositor austriaco (Campbell, 2001; Pauwels, Volterrani, Mariani \& Kostkiewics 2014).

Una de las explicaciones más relevantes sobre el supuesto del efecto Mozart, fue la identificación de un mejor estado de ánimo después de escuchar la pieza musical para realizar las actividades (Talero-Gutiérrez \& Saade-Lemus, 2018). Esto concuerda con lo propuesto por Kumar et al. (2016); Landay \& Harms (2018) y Seaborne \& Fiorella (2018), que señalan que escuchar música antes de estudiar ayuda a mejorar el estado de ánimo de los estudiantes, siempre y cuando la música sea preferida del sujeto. Sin embargo, Groot (2006), y Jäncke \& Sandmann (2010), Perham \& Vizard (2011), Perham \& Sykora (2012), Lehmann \& Seufert (2017), Custodio y Cano (2017), Schiller et al. (2018), Echaide et al. (2019), y Li et al. (2019), han reportado que la música preferida puede ser más disruptiva para la concentración en el estudio académico que la música no preferida o desconocida, como se ha señalado. Más tarde, Rausher \& Hinton (2010) involucraron el entrenamiento musical al efecto Mozart para sostener sus supuestos beneficios, sin embargo, el adiestramiento musical como se muestra más adelante, dista mucho del mero hecho de escuchar las piezas musicales para que por sí solas favorezcan el desarrollo cognitivo. En este sentido, se presentan a continuación, los efectos más sobresalientes del entrenamiento musical en el estudio y el aprendizaje.

\section{Hallazgos sobre los efectos del entrena- miento musical en el estudio y el aprendi- zaje}

Ekici \& Bilen (2010) indican que el entrenamiento musical adecuado no se trata solo de instruir a los alumnos en leer las partituras, o 
enseñar en colectivo a tocar o conocer un instrumento, sino como explican Yilmaz (2015) y Swaminathan, Schellenberg \& Khalil (2017), en instruir de manera preponderamente individual a los estudiantes, en un contexto teórico y práctico de la lectura y la producción musical de varios instrumentos; además, un adiestramiento musical adecuado no es esporádico, sino más bien continuo y perene, ya que la destreza musical requiere de muchas horas en práctica y repetición. En este sentido, Altenmüller (2002), identificó que los alumnos que estudian música en cursos que enfatizan en lecciones teóricas, muestran un menor desempeño que aquellos que poseen cursos de música que priorizan en experiencias para la representación musical mediante la ejecución de instrumentos y el canto. Por tanto, Kafol, Denac, Žnidaršič \& Zalar (2015), indican que los profesores de música más sobresalientes tienden a planear sus clases enfatizando directamente en la praxis, más que en un plan de estudios inclinado hacia el aspecto teórico.

Una de las bondades del entrenamiento musical es la práctica de la coordinación visuoespacial, auditiva y motora que exige con el adiestramiento, un nivel de precisión cada vez más complejo para la ejecución de piezas y cadencias melódicas más elaboradas (Schlaug, 2015). En este sentido, Justel y Díaz (2012), Graziano et al. (2016) y Sala \& Gobet (2017) identificaron que los alumnos que reciben entrenamiento musical adecuado desde jóvenes muestran una mayor capacidad de discriminación y asociación auditiva, visual y motora que aquellos alumnos no músicos o que recibieron una instrucción musical esporádica. Rose, Bartoli \& Heaton (2019), indican que la asociación auditiva, visual y motora en los músicos, sugiere una capacidad para vincular e imaginar sonidos que pueden ser interpretados de forma motora en el instrumento o en el canto. En este respecto, Wolf, Kopiez \& Platz (2018) indican que los músicos pueden imaginar visualmente los acordes y las cadencias musicales para construir secuencias melódicas creativas, incluso sin la presencia de sonido.

Por otra parte, Chiang et al. (2018) y Hardin et al. (2019), identificaron que los estudiantes que reciben entrenamiento musical adecuado, presentan una mayor habilidad para la distinción y diferenciación de tonos imperceptibles para los no músicos. Además, como indican Frank (2014) y Jicol et al. (2018), los músicos pueden discriminar con mayor facilidad los sonidos de los instrumentos en una pieza musical, enfocándose en la cadencia melódica de un solo instrumento, dentro de un conjunto de instrumentos que ejecutan simultáneamente.

En lo relativo al lenguaje y el entrenamiento musical, Rauscher, Shaw, Levine, Wright, Dennis \& Newcomb (2016), Habibi, Cahn, Damasio \& Damasio, H. (2016) indicaron que la habilidad sensitiva tonal de los alumnos músicos, puede ayudarles a distinguir con mayor facilidad la cadencia fonética del tono del habla, identificado con mayor precisión los cambios de humor en otras personas. Además, Rose et al. (2019) y Tierney, Rosen \& Dick (2020), observaron que los alumnos músicos muestran una mayor facilidad para la categorización de palabras de acuerdo con su significado emocional. En este sentido, Groot (2006) y Carrasco et al. (2016), argumentan que los estudiantes que reciben entrenamiento musical adecuado, presentan una mayor habilidad para diferenciar las relaciones tonales en la pronunciación de las palabras y las frases, favoreciendo la entonación en los discursos orales. Con base en estos hallazgos, Galicia y Zarzosa (2010) y 
Swaminathan, Schellenberg \& Venkatesan (2018), informaron una mayor habilidad de la conciencia fonológica de los alumnos con entrenamiento musical para la distinción de las vinculaciones de los segmentos fonológicos de las palabras (resultados similares también reportados por Anvari, Trainor, Woodside \& Levy, 2002; Zendel, West, Belleville \& Peretz, 2019; Vidal, Lousada \& Vigário, 2020).

El lenguaje es una habilidad transversal que impacta en todas las asignaturas escolares, por lo que diversos estudios han observado las implicaciones que genera el entrenamiento musical en el desarrollo del lenguaje (Brown, Benedett \& Armistead, 2010). En este respecto, Talamini et al. (2018) identificaron una mayor habilidad de los alumnos con entrenamiento musical para la percepción auditiva de silabas fonológicas en la constitución del vocabulario, durante el aprendizaje de un segundo idioma, además, estos autores (Talamini et al., 2018), observaron que los estudiantes músicos se mostraron más motivados durante las sesiones de conversación práctica de lenguas extranjeras. Ho, Cheung \& Chan (2003) por su parte, realizaron un estudio longitudinal donde observaron que los alumnos que recibían entrenamiento musical adecuado, retenían mejor el vocabulario aprendido que los no músicos. Después de un año de seguimiento, Ho et al. (2003), indicaron que los estudiantes que continuaron con el entrenamiento musical siguieron demostrando la habilidad de retención de vocabulario, sin embargo, no fue así en aquellos alumnos que dejaron el entrenamiento musical. Resultados similares también fueron reportados por Degé, Wehrum, Stark \& Schwarzer (2011) y Herrera, Hernández, Lorenzo y Ropp (2014).

Por otra parte, en relación a los efectos del entrenamiento musical y su impacto en el área de matemáticas. Matute (2012) y Hardin et al. (2019), indicaron que los alumnos que reciben entrenamiento musical, presentan mejores puntajes en pruebas de memoria que los no músicos, sugiriendo que la producción musical demanda de la memorización del procesamiento psicomotriz para la creación y soporte de largas secuencias imaginativas de sonidos armónicos organizados en el tiempo, que después son ejecutados en el instrumento.

En este sentido, Holmes \& Hallam (2017) señalan que la retención de secuencias de información en la memoria demandadas por la producción musical, puede favorecer el sostenimiento de datos para realizar tareas de cálculo mental. Estos autores (Holmes \& Hallam, 2017), identificaron mejores puntajes en alumnos músicos sobre ejercicios de matemáticas en clase que los estudiantes no músicos. Asimismo, Schlaug (2015) y Graziano et al. (2016), también informaron que los alumnos con entrenamiento musical adecuado, presentaron mejores puntajes en pruebas de cálculo mental que los no músicos.

Por lo general, las bases del cálculo mental radican en operaciones simultáneas y secuenciales de adición, sustracción, multiplicación y división de datos, como métodos estándar que demandan la retención de información (Hoch \& Tillmann, 2012). Estos datos para el cálculo matemático además de precisar de la memoria, contienen patrones de organización y tratamiento, lo que también sucede en la música, a pesar de la superposición armónica de diferentes cadencias melódicas simultáneas (Willis, 2016). En este sentido, Godoy \& Jorgensen (2001), McPherson (2005) y Matas (2013), indicaron que las personas que reciben entrenamiento musical adecuado, presentan mejores habilidades para encontrar patrones de organización operacionales en cálculos matemáticos, que los no 
músicos. Otros estudios (Bahr \& Christensen, 2000; Schmithorst \& Holland, 2004; Helmrich, 2010; Cranmore \& Tunks, 2015; Murray, 2016), también han reportado mejores puntajes en pruebas de matemáticas por parte de personas con entrenamiento musical.

Por otro lado, Foster \& Jenkins (2017) encontraron que los alumnos con entrenamiento musical, presentan una mayor proyección emocional para la representación de las artes escénicas. En este respecto, Hardiman (2016), encontró una mayor habilidad en los estudiantes músicos para improvisar en las artes escénicas como el teatro, que los alumnos no músicos. Por su parte, Fogerald, Winner, Norton \& Schlaug, (2008) indicaron que los estudiantes con entrenamiento musical presentaron mejores habilidades para expresar gestos y actitudes sentimentales durante obras de representación teatral, que los no músicos.

Hyde, Lerch, Norton, Forgerad, Winner, Evans \& Schlaug (2009) y Carrasco et al. (2016), indican que la sensibilidad emocional y la percepción tonal en los músicos puede mejorar la expresión oral en las artes escénicas, aunque, por otro lado, Loffing, Hagemann, Strauss \& MacMahon (2016), observaron mejores habilidades motoras finas en los estudiantes músicos para la elaboración de trabajos de manualidades en clase. En este respecto. Limb y Braun (2008) y Hardiman (2016), identificaron que los alumnos músicos, modularon con mayor facilidad el tono de voz en los discursos y en las expresiones de obras de representación teatral. Además, Corrigall, Schellenberg \& Misura (2013), observaron que los estudiantes músicos mostraron un mejor control emocional para la interpretación teatral que los no músicos, incluso Kraus \& Chandrasekaran (2010) y White, Hutka, Williamsn \& Moreno (2013), indica- ron que los alumnos con entrenamiento musical, presentan un mejor dominio emocional ante el pánico escénico en artes representacionales como el teatro, respecto a los estudiantes no músicos.

Por otro lado, Patston, Hogg \& Tippett (2007), Snowman (2010) e Iuşcă (2014), reportaron que los músicos que ejecutan habitualmente instrumentos con ambas extremidades superiores finas, muestran una mayor preponderancia motora bilateral, que aquellos músicos que solo ejecutan instrumentos con una mano; no obstante, por otra parte, Martins, Neves, Rodrigues, Vasconcelos \& Castro (2018), identificaron que tanto alumnos músicos bilaterales o no, mostraron mejores puntajes que los no músicos en pruebas de movimiento de precisión, que involucraban tareas de lanzamiento, seguimiento y agarre de objetos en el aire.

Al respecto, Simpkins, Vest \& Becnel (2010) y Rauscher et al. (2016), indican que los estudiantes que reciben entrenamiento musical adecuado, muestran una mayor habilidad para la orientación en el espacio, sugiriendo como explican Franklin, Sledge, Yip \& Jonides (2008), que los músicos pueden imaginar la visualización de la superposición armónica de una cadencia musical al tiempo que deben plasmarla con precisión motora en el espacio de ejecución física del instrumento. Esta correlación visuomotora puede impactar en el entendimiento de las relaciones espaciales de otros objetos. En este respecto, Schlaug (2015) y Jicol et al. (2018), reportaron una mejor coordinación en los estudiantes músicos para la percepción consciente del contexto, durante la ejecución motora espacial precisa de los acordes y las escalas en los instrumentos. 


\section{Conclusiones}

La evidencia sugiere que la música de fondo preferida puede causar un incremento emocional en el estudiante que genere una mayor cantidad de imaginerías mentales y recuerdos emocionales que perturben la concentración en el estudio académico. En este sentido, diversos estudios (Neville et al., 2008; Anderson \& Fuller, 2010; Johansson et al., 2012; Cauchard et al., 2012; Christopher \& Shelton, 2017; Khan et al., 2018; Zhang et al., 2018), muestran que la música de fondo en el estudio académico aumenta la frecuencia en el desvío de la mirada del material de estudio, haciendo la atención más intermitente, es decir, que el sujeto coloca atención en el material de estudio con momentos sutiles de atención consciente hacia la pieza musical, haciendo más lento el proceso de estudio académico. En este respecto, diversas investigaciones (Tervaniemi et al., 2006; Salamé \& Baddeley, 2007; Trost, Ethofer, Zentner \& Vuilleumier, 2012; Perham \& Currie, 2014; Kang \& Lakshmanan, 2017; Schiller et al., 2018; Koelsch et al., 2019), han mostrado que música con lírica interrumpe con mayor frecuencia el estudio académico que la música instrumental, dado el fin comunicativo de las letras y el mensaje prosódico de las canciones. Por otro lado, otros estudios (Furnham \& Allas, 1999; Hallam et al., 2010; Jäncke \& Sandmann, 2010; Kumar et al., 2016) han reportado que la música a ritmo rápido perturba más la concentración en el estudio académico que la música con ritmo lento. Sin embargo, a pesar de los efectos sobre la injerencia de la música de fondo en el estudio, la mayoría de los estudios (Oswald et al., 2000; Pool et al., 2003; Sweller, 2003; Salamé \& Baddeley, 2007; Neville et al., 2008; Furnham \& Strbac, 2010; Anderson \& Fuller, 2010; Moreno \& Mayer, 2010; Perham \& Vizard, 2011; Dobbs et al.,
2011; Penagos-Corzo y De La Fuente, 2012; Perham \& Sykora, 2012; Johansson et al., 2012; Cauchard et al., 2012; Reynolds et al., 2014; Flanagan \& Alfonso, 2016; Custodio y Cano, 2017; Lehmann \& Seufert, 2017; Kang \& Lakshmanan, 2017; Christopher \& Shelton, 2017; Lehmann et al., 2018; Khan et al., 2018; Schiller et al., 2018), han mostrado que la condición de silencio resulta menos perturbadora que la presencia de música de fondo (instrumental o con lírica; con ritmo lento o rápido) $\mathrm{y}$ de ruido (conversaciones de personas, ruido blanco o rosa) en la concentración para el estudio académico.

Por otra parte, se observó que los estudios sobre los efectos del entrenamiento musical en el estudio académico y el aprendizaje son consistentes en el favorecimiento de cuatro áreas académicas y cognitivas esenciales: el lenguaje, el cálculo mental, la representación de artes escénicas y la orientación espacial. Si bien se apreció que los efectos del entrenamiento musical son específicos para algunos rubros de estas áreas, cabe indicar que estas habilidades son trasversales e importantes para la educación formal.

Entre los hallazgos que reportan los estudios (Anvari et al., 2002; Ho et al., 2003; Groot, 2006; Galicia y Zarzosa, 2010; Brown et al., 2010; Degé et al., 2011; Rauscher et al., 2016; Carrasco et al., 2016; Habibi et al., 2016; Jicol et al., 2018; Swaminathan et al., 2018; Talamini et al., 2018; Hardin et al., 2019; Rose et al., 2019; Tierney et al., 2020; Vidal et al., 2020) que muestran efectos benéficos del entrenamiento musical para el área de lenguaje, se apreció la facilidad en la sensibilidad tonal para identificar la cadencia fonética del tono del habla en otras personas y una mejor facilidad para la entonación durante la pronunciación del discurso oral. Además, se apercibió una mejor habilidad en los alumnos músicos para la distinción de las re- 
laciones entre los segmentos fonológicos de la constitución de las palabras. Por otro lado, entre los hallazgos que reportan los estudios (Bahr \& Christensen, 2000; Godoy \& Jorgensen, 2001; Schmithorst \& Holland, 2004; Schlaug, Norton, Overy \& Winner, 2005; McPherson, 2005; Helmrich, 2010; Matute, 2012; Hoch \& Tillmann, 2012; Matas, 2013; Cranmore \& Tunks, 2015; Willis, 2016; Murray, 2016; Graziano et al., 2016; Holmes \& Hallam, 2017; Hardin et al., 2019) sobre el área de matemáticas, se apreció que el entrenamiento musical favoreció la retención de secuencias de datos numéricos para organizarlos durante el cálculo mental. Además, se reportó una mejor habilidad en los estudiantes músicos para identificar patrones de organización operacionales en cálculos matemáticos.

Sobre el área de la representación escénica, se distinguió de los hallazgos de diversos estudios (Fogerald et al., 2008; Limb y Braun, 2008; Hyde et al., 2009; Kraus \& Chandrasekaran, 2010; Corrigall et al., 2013; White et al., 2013; Hardiman, 2016; Loffing et al., 2016; Foster \& Jenkins, 2017), que los estudiantes con entrenamiento musical muestran una mayor habilidad para la proyección emocional durante el discurso en representaciones teatrales, así como una mayor facilidad para improvisar y expresar gestos y actitudes sentimentales. Por otra parte, en cuanto a los hallazgos de los estudios (Franklin et al., 2008; Snowman, 2010; Simpkins et al., 2010; Iuşcă, 2014; Schlaug, 2015; Rauscher et al., 2016; Jicol et al., 2018; Martins et al., 2018) sobre el tema de la orientación espacial, se apreció que el entrenamiento musical favoreció los movimientos de precisión mediante una correlación visuomotora para tareas de lanzamiento, seguimiento y agarre de objetos.

Estas contribuciones apoyan al respaldo de la hipótesis de trabajo que dictó: el uso de la música de fondo es más disruptiva para la concentración que la condición de silencio durante el estudio autónomo académico, y además, el entrenamiento musical adecuado produce mayores ventajas cognitivas que el hecho de escuchar música antes o durante el estudio académico. Es necesario, continuar incentivando el auge de investigaciones que permitan un mayor entendimiento sobre la tendencia y la consistencia de los estudios que se han centrado en conocer los efectos de la música de fondo en el estudio académico, y sobre los efectos cognitivos del entrenamiento musical en otras circunstancias que permitan ampliar el panorama de entendimiento sobre las diferencias correlacionales entre la tipología y la duración del entrenamiento musical, observando sus efectos a corto, mediano y largo plazo respecto al aprendizaje de las diferentes áreas curriculares, considerando cada uno de los distintos niveles educativos.

\section{Referencias}

Akelma, H., Altinsoy, S, Arslan, M., \& Ergil, J. (2020). Effect of Favorite Music on Postoperative Anxiety and Pain. Anaesthesist, 69(3), 198204. doi: 10.1007/s00101-020-00731-8

Aljanaki, A., Wiering, F., \& Veltkamp, R. (2016). Studying emotion induced by music through a crowdsourcing game. Information Proccesing \& Management, 52(1), 115-158. doi: 10.1016/ j.ipm.2015.03.004

Allen, E., Burton, P., Olman, C., \& Oxenham, A. (2017). Representations of Pitch and Timbre Variation in Human Auditory Cortex. Journal of Neuroscience, 37(5), 1284-1293. doi: 10.1523/ JNEUROSCI.2336-16.2016

Altenmüller, E. (2002). Neurología de la percepción musical. Mente y Cerebro, 2002(1), 48-55. Recuperado de: https://dialnet.unirioja.es/servlet/ articulo? codigo $=2809116$

Anderson, S., Henke, J., McLaughlin, M., Ripp, M., \& Tuffs, P. (2000). Using Background Music to Enhance Memory and Improve Learning. Washington: Institute of Education Sciences. Recuperado de: http://www.eric.ed.gov/ ERICWebPortal/search/detailminin.jsp? nfpb $=$ true 
Anderson, S., \& Fuller, G. (2010). Effect of music on reading comprehension of junior high school students. School Psychology Quarterly, 25(3), 178-187. doi: 10.1037/a0021213

Angulo-Perkins, A., Aubé, W., Peretz, I., Barrios, F., Armony, J., \& Concha, L. (2014). Music listening engages specific cortical regions within the temporal lobes: Differences between musicians and non-musicians. Cortex, 59(1), 126-137. doi: 10.1016/j.cortex.2014.07.013

Anvari, S., Trainor, L., Woodside, J., \& Levy, B. (2002). Relations among musical skills, phonological processing, and early reading ability in preschool children. Journal of Experimental Child Psychology, 83(1), 111-130. doi: 10.1016/S0022-0965(02)00124-8

Baddeley, A. (2007). Working memory. Oxford: Oxford University Press.

Bahr, N., \& Christensen, C. (2000). Inter-domain transfer between mathemetical skills and musicianship. Journal of Structural Learning \& Intelligent Systems, 14(3), 187-197. Recuperado de: https://psycnet.apa.org/record/2000-03755-001

Banbury, S., \& Berry, D. (2010). Office noise and employee concentration: Identifying causes of disruption and potential improvements. Ergonomics, $48(1), \quad 25-37 . \quad$ doi: $10.1080 / 00140130412331311390$

Bashwiner, D., \& Bacon. D. (2019). musical creativity and the motor system. Current Opinion in Behavioral Sciences, 27(1), 146-153. doi: 10.1016/j.cobeha.2018.12.005

Bellur, S., Nowak, K., \& Hull, K. (2015). Make it our time: In class multitaskers have lower academic performance. Computers in human Behavior, 53 (1), 63-70. doi: 10.1016/j.chb.2015.06.027

Ben-Nathan, M., Salti, M., \& Algom, D. (2019). The many faces of music: Attending to music and delight in the same music are governed by different rules of processing. Acta Psychologica, 200(1), 102-105. doi: 10.1016/ j.actpsy.2019.102949

Biasuti, M. (2019). Self-assessing music therapy: The validity and reliability of the music therapy practice scale. The Arts in Psychotherapy, 63 (1), 40-45. doi: 10.1016/j.aip.2019.03.006

Bigliassi, M., Karageorghis, C., Bishop, D., Nowicky, A., \& Wright, M. (2018). Cerebral effects of music during isometric exercise: An fMRI study. International Journal of Psychophysiology, 133(1), 131-139. doi: 10.1016/ j.ijpsycho.2018.07.475

Bloor, A. (2009). The rhythm's gonna get ya' - background music in primary classrooms and its effect on behaviour and attainment. Emotional and Behavioural Difficulties, 14(4), 261-274. doi: 10.1080/13632750903303070

Boghdady, M., \& Ewalds-Kvist, B. (2020). The influence of music on the surgical task performance. International Journal of Surgery, 73(1), 101112. doi: 10.1016/j.jpsu.2019.11.012

Brown, E., Benedett, B., \& Armistead, E. (2010). Arts enrichment and school readiness for children at risk. Early Childhood Research Quarterly, 25 (1), 112-124. doi: 10.1016/j.ecresq.2009.07.008

Burunat, I., Alluri, V., Toiviainen, P., Numminen, J., \& Brattico, E. (2014). Dynamics of brain activity underlying working memory for music in a naturalistic condition. Cortex, 57(1), 254-269. doi: 10.1016/j.cortex.2014.04.012

Butera, I. (2015). From Notes to Vowels: Neural Correlations between Musical Training and Speech Processing. Journal of Neuroscience, 35(22), 8379-8381. doi: 10.1523/JNEUROSCI.110215.2015

Calderwood, C., Ackerman, P., \& Conklin, E. (2014). What else do college students "do" while studying? An investigation of multitasking. Computers \& Education, 75(1), 19-29. doi: 10.1016/ j.compedu.2014.02.004

Campbell, D. (2001). The Mozart effects. New York: Harper Collins Publishers.

Cantú, D., Lera, J., y Baca, J. (2017). Especialización hemisférica y estudios sobre lateralidad. Revista de Psicología y Ciencias del Comportamiento, 8(2), 6-50. doi: 10.29365/rpcc.20171229-58

Carrasco, A., Gustems, J., y Calderón, C. (2016). Ritmo y procesamiento temporal. Aportaciones de Jaques-Dalcroze al lenguaje musical. Magister, 28(1), 35-41. doi: 10.1016/j.magis.2016.06.003

Cauchard, F., Cane, J., \& Weger, U. W. (2012). Influence of background speech and music in interrupted reading: An eye-tracking study. Applied Cognitive Psychology, 26(3), 381-390. Doi: 10.1002/acp.1837

Charif, M., Mitrofan, L., \& Gâtej, E. (2014). The Influence of Relaxation Music on Abstract Visual Short-Term Memory Retrieval Task at Young Students at Psychology. Procedia, 127(1), 852857. doi: 10.1016/j.sbspro.2014.03.367

Chiang, J., Rosenberg, M., Bufford, C., Stephens, D., Lysy, A., \& Monti, M. (2018). The language of music: Common neural codes for structured sequences in music and natural language. Brain and Lenguage, 185(1), 30-37. doi: 10.1016/ j.bandl.2018.07.003

Chiat, L., \& Fung, L. (2012). Importance of Music Learning and Musicality in Rhythmic Gymnas- 
tics. Procedia, 46(1), 3202-3208. doi: 10.1016/ j.sbspro.2012.06.037

Christopher, E., \& Shelton, J. (2017). Individual Differences in Working Memory Predict the Effect of Music on Student Performance. Journal of Applied Research in Memory and Cognition, 6 (2), 167-173. doi: 10.1016/j.jarmac.2017.01.012

Corrigall, K., Schellenberg, G., \& Misura, N. (2013). Music training, cognition, and personality. Frontiers in Psychology, 4(19), 221-223. doi: 10.3389/fpsyg.2013.00222

Cranmore, J., \& Tunks, J. (2015). Brain Research on the Study of Music and Mathematics. Journal of Mathematics Education, 8(2), 139-157. Recuperado de http://educationforatoz.com/ images/2015_Jeff_Cranmore.pdf

Črnčec, R., Wilson, S., \& Prior, M. (2006). The Cognitive and Academic Benefits of Music to Children: Facts and fiction. Educational Psychology: An International Journal of Experimental Educational Psychology, 26(4), 579-594. doi: $10.1080 / 01443410500342542$

Custodio, N., y Cano, M. (2017). Efectos de la música sobre las funciones cognitivas. Revista de Neuro-Psiquiatría, 80(1), 61-71. Recuperado en: https://www.redalyc.org/ pdf/3720/372050405008.pdf

Damasio, A. (2006). Descartes' Error: Emotion, Reason, and the Human Brain. New York: Pan Macmillan.

Davidson, Ch., \& Powell, L (2015). The Effects of Easy-Listening Background Music on the OnTask-Performance of Fifth-Grade Children. The Journal of Educational Research, 80(1), 29-33. doi: 10.1080/00220671.1986.10885717

Daykin, N., Mansfield, L., Meads, C., Julier, G., Tomlinson, A., Payne, A... \& Victor, C. (2018). What Works for Wellbeing? A Systematic Review of Wellbeing Outcomes for Music and Singing in Adults. Perspectives in Public Health, 138(1), 39-46. doi: $10.1177 / 1757913917740391$

Degé, F., Wehrum, S., Stark, R., \& Schwarzer, G. (2011). The influence of two years of school music training in secondary school on visual and auditory memory. European Journal of Developmental Psychology, 81(5), 608-623. doi: 10.1080/17405629.2011.590668

Dobbs, S., Furnham, A., \& McClelland, A. (2011). The effect of background music and noise on the cognitive test performance of introverts and extraverts. Applied Cognitive Psychology, 25 (2), 2-22. doi: 10.1002/acp.1692
Dosseville, F., Laborde, S., \& Scelles, N. (2012). Music during lectures: Will students learn better? Learning and Individual Differences, 22(2), 258 -262. doi: 10.1016/j.lindif.2011.10.004

Doyle, M., \& Furnham, A. (2012). The distracting effects of music on the cognitive test performance of creative and non-creative individuals. Thinking Skills and Creativity, 7(1), 1-7. doi: 10.1016/j.tsc.2011.09.002

Echaide, C., Del Río, D., \& Pacios, J. (2019). The differential effect of background music on memory for verbal and visuospatial information. The Journal of General Psychology, 146(4), 443458. doi: 0.1080/00221309.2019.1602023

Ekici, T., \& Bilen, S. (2010). A study of need analysis on individual voice education course for music teacher training. Procedia, 2(2), 4878-4886. doi: 10.1016/j.sbspro.2010.03.788

Engle, R., \& Kane, M. (2003). Executive Attention, Working Memory Capacity, and a Two-Factor Theory of Cognitive Control. Psychology of Learning and Motivation, 44(1), 145-199. doi: 10.1016/S0079-7421(03)44005-X

Ettenberger, M., \& Beltrán, Y. (2018). Music therapy song writing with mothers of preterm babies in the Neonatal Intensive Care Unit. The Arts in Psychotherapy, 58(1), 42-52. doi: 10.1016/ j.aip.2018.03.001

Feierabend, J. (2010). Music in Early Childhood. Design for Arts in Education, 91(6), 15-20. doi: 10.1080/07320973.1990.9934833

Flanagan, D., \& Alfonso, V. (2016). WJ IV Clinical Use and Interpretation. New York: Academic Press.

Fogerald, M., Winner, E., Norton, A., \& Schlaug, G. (2008). Practicing a Musical Instrument in Childhood is Associated with Enhanced Verbal Ability and Nonverbal Reasoning. PLoS One, 3 (10), 3566-3570. doi: 10.1371/ journal.pone.0003566

Foster, E., \& Jenkins, K. (2017). Does Participation in Music and Performing Arts Influence Child Development? American Educational Research Journal, 54(3), 63-73. doi: 10.3102/0002831217701830

Frank, G. (2014). Music to My Brain: Could Music Training Be Used to Improve Adolescent Brain Development? Journal of American Academy of Child and Adolescent Psychiatry, 53(11), 1147-1149. doi: 10.1016/j.jaac.2014.07.016

Franklin, M., Sledge, K., Yip, C., \& Jonides, J. (2008). The effects of musical training on verbal memory. Psychology of Music, 36(3), 353-365. doi: $10.1177 / 0305735607086044$ 
Furnham A., \& Allas, K. (1999). The influence of musical distraction of varying complexity on the cognitive performance of extroverts and introverts. European Journal of Personality, 13(1), 27-38. Recuperado de: https://www.gwern.net/ docs/music-distraction/1999-furnham-2.pdf

Furnham, A., \& Strbac, L. (2010). Music is as distracting as noise: the differential distraction of background music and noise on the cognitive test performance of introverts and extraverts. Ergonomics, 45(3), 203-217. doi: $10.1080 / 00140130210121932$

Galicia, I., y Zarzosa, L. (2010). La presencia de las actividades musicales en los programas educativos y su influencia en la conciencia fonológica. Perfiles Educativos, 36(144), 157-161. doi: 10.1016/S0185-2698(14)70629-4

Galińska, E. (2015). Music Therapy in Neurological Rehabilitation Settings. Psychiatria Polska, 49 (4), 835-846. doi: 10.12740/pp/25557.

Gaser, C., \& Schlaug, G. (2003). Brain structures differ between musicians and non-musicians. Journal of Neuroscience, 23(27), 9240-9245. doi: 10.1523/JNEUROSCI.23-27-09240.2003

Geretsegger, M., Elefant, C., Mössler, K., \& Gold, C. (2014). Music Therapy for People with Autism Spectrum Disorder. Cochrane Database of System Reviews, 2016(6), 112-114. doi: 10.1002/14651858.CD004381.pub3

Godoy, R., \& Jorgensen, H. (2001). Musical imagery. London: Taylor \& Francis.

Graziano, A., Peterson, M., \& Shaw, G. (2016). Enhanced learning of proportional math through music training and spatial-temporal training. Neurological Research, 21(2), 139-152. doi: 10.1080/01616412.1999.11740910

Groot, A. (2006). Effects of stimulus characteristics and background music on foreign language vocabulary learning and forgetting. Language Learning, 56(3), 17-23. doi: 10.1111/j.14679922.2006.00374.x

Guerra, M., \& Muñoz de Rodríguez, L. (2013). Attachment expressions and behaviors in mothers and their newborn hospitalized in a Neonatal Intensive Care Unit. Enfermería Universitaria, 10(3), 84-91. doi: 10.1016/s1665-7063(13)72633-2

Gutgsell, K., Schluchter, M., Margevicius, S., DeGolia, P., McLaughlin, B., Harris, M., ... \& Wiencek, C. (2013). Music Therapy Reduces Pain in Palliative Care Patients. Journal of Pain and Symp- tom Management, 45(5), 822-831. doi: 10.1016/ j.jpainsymman.2012.05.008

Habibi, A., Cahn, B., Damasio, A., \& Damasio, H. (2016). Neural correlates of accelerated auditory processing in children engaged in music training. Developmental Cognitive Neuroscience, 21(1), 1-14. doi: 0.1016/j.den.2016.04.003

Habibi, A., Damasio, A., Ilari, B., Joshi, A., Leahy, R, Haldar, J... \& Damasio, H. (2018). Childhood Music Training Induces Change in Micro and Macroscopic Brain Structure: Results from a Longitudinal Study. Cerebral Cortex, 28(12), 4336-4347. doi: 10.1093/cercor/bhx286

Hafteck, L. (2006). Music and Language Development in Early Childhood: Integrating Past Research in the Two Domains. Early Child Development and Care, 130(1), 85-97. doi: 10.1080/0300443971300109

Hallam, S., Price, J., \& Katsarou, G. (2010). The Effects of Background Music on Primary School Pupils' Task Performance. Educational Studies, 28(2), 111-122. doi: $10.1080 / 03055690220124551$

Hallyday, A. (2019). Bridging music and organizational psychology: Everyday music uses and preferences and the prediction of organizational behaviour. Personality and Individual Differences, 139(1), 263-276. doi: 10.1016/ j.paid.2018.10.042

Hardiman, M. (2016). Education and the arts: educating every child in the spirit of inquiry and joy. Creative Education, 7(1), 1913-1928. doi: 10.4236/ce.2016.714194

Hardin, E., Sammler, D., Henry, M., Large, E., \& Kotz, S. (2019). Cortical tracking of rhythm in music and speech. NeuroImage, 185(1), 96-101. doi: $0.1016 /$ j.neuroimage.2018.10.037

Helmrich, B. (2010). Window of Opportunity? Adolescence, Music, and Algebra. Psychology, 25(4), 557-577. 10.1177/0743558410366594

Herrera, L., Hernández, M., Lorenzo, O., y Ropp, C. (2014). Influencia del entrenamiento musical en el desarrollo cognitivo y lingüístico de niños de 3 a 4 años. Revista de Psicodidáctica, 19(2), 367-386. Recuperado de: https:// www.redalyc.org/pdf/175/17531400007.pdf

Ho, Y., Cheung, M., \& Chan, A. (2003). Music training improves verbal but not visual memory: Cross-sectional and longitudinal explorations in 
children. Neuropsychology, 17(3), 439-450. doi: 10.1037/0894-4105.17.3.439

Hoch, L., \& Tillmann, N. (2012). Shared structural and temporal integration resources for music and arithmetic processing. Acta Psychologica, 140 (3), 230-235. doi: 10.1016/j.actpsy.2012.03.008

Holmes, S., \& Hallam, S. (2017). The impact of participation in music on learning mathematics. London Review of Education, 15(2), 425-438. doi: 10.18546/LRE.15.3.07

Hsing-Chi, A., \& Oh, J. (2020). Interacting with background music engages E-Customers more: The impact of interactive music on consumer perception and behavioral intention. Journal of Retailing and Consumer Services, 54(1), 101109. doi: 10.1016/j.jretconser.2019.101928

Hyde, K., Lerch, J., Norton, A., Forgerad, M., Winner, E., Evans, A., \& Schlaug, G. (2009). Musical Training Shapes Structural Brain Development. Journal of Neuroscience, 29(10), 3019-3025. doi: 10.1523/jneurosci.5118-08.2009

Hynes, N., \& Manson, S. (2016). The sound of silence: Why music in supermarkets is just a distraction. Journal of Retailing and Consumer Services, 28 (1), 171-178. doi: 10.1016/ j.jretconser.2015.10.001

Iuşcă, D. (2014). Brain Hemisphericity and Music Performance Level in Undergraduate Music Students and Philharmonic Musicians. Procedia, 142(1), 59-63. doi: 10.1016/ j.sbspro.2014.07.588

Jäncke, L., \& Sandmann, P. (2010). Music listening while you learn: No influence of background music on verbal Learning. Behavioral and Brain Functions, 6(3), 6-12. doi: 10.1186/1744-90816-3

Jeong, E., \& Ryu, H. (2016). Nonverbal auditory working memory: Can music indicate the capacity? Brain and Cognition, 105(1), 9-21. doi: 10.1016/j.bandc.2016.03.003

Jicol, C., Proulx, M., Pollick, F., \& Pretini, K. (2018). Long-term Music Training Modulates the Recalibration of Audiovisual Simultaneity. Experimental Brain Research, 236(7), 1869-1880. doi: 10.1007/s00221-018-5269-4.

Johansen-Berg, H. (2001). Music to your baby's ears. Trends in Cognitive Sciencies, 5(9), 377-378. doi: 10.1016/S1364-6613(00)01761-7
Johansson, R., Holmqvist, K., Mossberg, F., \& Lindgren, M. (2012). Eye movements and reading comprehension while listening to preferred and non-preferred study music. Psychology of $\mathrm{Mu}$ sic, $\quad 40(3), \quad 339-356 . \quad$ doi: $10.1177 / 0305735610387777$

Judd, T. (2014). Making sense of multitasking: The role of Facebook. Computers \& Education, 70 (1), 194-202. doi: 10.1016/ j.compedu.2013.08.013

Justel, M., y Díaz, V. (2012). Plasticidad cerebral: participación del entrenamiento musical. Konrad Lorenz Fundación Universitaria, 19(2), 4-9. doi: 10.14349/sumapsi2012.1234

Kaarlela-Tuomaala, A., Helenius, R., Keskinen, E., \& Hongisto, V. (2009). Effects of acoustic environment on work in private office rooms and open-plan offices. Longitudinal study during relocation. Ergonomics, 52(11), 1433-1444. doi: 10.1080/00140130903154579

Kafol, B., Denac, O., Žnidaršič, J., \& Zalar, K. (2015). Analysis of Music Education Objectives in Learning Domains. Procedia, 186(1), 95-104. doi: $10.1016 /$ j.sbspro.2015.04.069

Kang, E., \& Lakshmanan, A. (2017). Role of executive attention in consumer learning with background music. Journal of Consumer Psychology, 27(1), 35-48. doi: 10.1016/j.jcps.2016.03.003

Khan, S., Kitsis, M., Golovyan, D., Wang, S., Chlan, L., Boustani, M., \& Khan, B. (2018). Effects of music intervention on inflammatory markers in critically ill and post-operative patients: A systematic review of the literature. Heart \& Lung, 47(5), 489-496. doi: 10.1016/ j.hrtlng.2018.05.015

Kim, C., Lee, S., Kim, J., Seol, J., Yi, S., \& Chung, C. (2014). Melody effects on ERANm elicited by harmonic irregularity in musical syntax. Brain Research, 1563(1), 36-45. doi: 10.1016/ j.brainres.2014.02.045

Kipnis, G., Tabak, N., \& Koton, S. (2016). Background Music Playback in the Preoperative Setting: Does It Reduce the Level of Preoperative Anxiety Among Candidates for Elective Surgery? Journal of PeriA nesthesia Nursing, 31(3), 209-216. doi: 10.1016/j.jopan.2014.05.015

Koelsch, S., Vuust, P., y Fritson, K. (2019). Predictive Processes and the Peculiar Case of Music. Trends in Cogntive Sciences, 23(1), 63-77. doi: 110.1016/j.tics.2018.10.006

Kraus, N., \& Chandrasekaran, N. (2010). Music training for the development of auditory skills. $\mathrm{Na}$ - 
ture Reviews, 11(1), 599-602. doi: 10.1038/ nrn2882

Kumar, N., Wajidi, M., Chian, Y., Vishroothi, S., Ravindra, S., \& Aithal, A. (2016). The effect of listening to music on concentration and academic performance of the student: Cross-sectional study on medical undergraduate students. Research Journal of Pharmaceutical, Biological and Chemical Sciences, 7(6), 1190-1195. Recuperado en: https://manipal.pure.elsevier.com/en/ publications/the-effect-of-listening-to-music-on -concentration-and-academic-pe

Lamb, S., \& Gregory, A. (2006). The Relationship between Music and Reading in Beginning Readers. Educational Psychology: An International Journal of Experimental Educational Psychology, 13(1), 19-27. doi: 10.1080/0144341930130103

Limb, C., \& Braun, A. (2008). Neural Substrates of Spontaneous Musical Performance: An fMRI Study of Jazz Improvisation. PLoS One, 3(2), 1979-1985. doi: 10.1371/journal.pone.0001679

Landay, K., \& Harms, P. (2018). Whistle while you work. A review of the effects of music in the workplace. Human Resource Management Review, (In Press). doi: 10.1016/ j.hrmr.2018.06.003

Lehmann, J., \& Seufert, T. (2017). The Influence of Background Music on Learning in the Light of Different Theoretical Perspectives and the Role of Working Memory Capacity. Frontiers in Psychology, 8(1902), 7-18. doi: 10.3389/ fpsyg.2017.01902

Lehmann, J., Hamm, V., \& Seufert, T. (2018). The influence of background music on learners with varying extraversion: Seductive detail or beneficial effect? Applied Cognitive Psychology, 33 (1), 85-94. doi: 10.1002/acp.3509

Li, C., Chen, T., \& Tsai, Ch. (2019). Music enhances activity in the hypothalamus, brainstem, and anterior cerebellum during script-driven imagery of affective scenes. Neuropsychologia, (In Press). doi: 10.1016/ j.neuropsychologia.2019.04.014

Loffing, F., Hagemann, N., Strauss, B., \& MacMahon, C. (2016). Laterality in sports. Theories and applications. New York: Academic Press.

Lozano, L. y Lozano, A. (2007). La influencia de la música en el aprendizaje. Mérida: ITESM.

Manes, F., y Niro, M. (2014). Usar el cerebro. Buenos Aires: Paidós.

Martins, M., Neves, L., Rodrigues, P., Vasconcelos, O., \& Castro, S. (2018). Orff-Based Music
Training Enhances Children's Manual Dexterity and Bimanual Coordination. Frontiers of Psychology, 9(1), 2616-2619. doi: 10.3389/ fpsyg.2018.02616

Matas, A. (2013). Efecto favorable del tempo musical lento en el cálculo mental aritmético. Revista de Investigación y Cultura, 2(1), 10-19. Recuperado en: https:/www.redalyc.org/ pdf/5217/521752180003.pdf

Matute, E. (2012). Tendencias actuales de las neurociencias cognitivas. México: Manual Moderno.

McClurkin, S., \& Smith, C. (2016). The Duration of Self-Selected Music Needed to Reduce Preoperative Anxiety. Journal of PeriAnesthesia Nursing, 31(3), 196-208. doi: 10.1016/ j.jopan.2014.05.017

McKetton, L., DeSimone, K., \& Schneider, K. (2019). Larger Auditory Cortical Area and Broader Frequency Tuning Underlie Absolute Pitch. Journal of Neuroscience, 39(15), 2930-2937. doi: 10.1523/jneurosci.1532-18.2019

McPherson, G. (2005). From child to musician: skill development during the beginning stages of learning an instrument. Psychology of Music, 33(1), 5-35. doi: 10.1177-0305735605048012

Miché, M. (2002). Weaving music into young minds. New York: Delmar Thomson Learning.

Michel, A., Braumann, C., \& Gayer, L. (2017). Thank you for the music - or not? The effects of instore music in service settings. Journal of Retailing and Consumer Services, 36(1), 21-32. doi: 10.1016/j.jretconser.2016.12.008

Mitchell, H. (2018). Music students' perceptions of experiential learning at the moot audition. $\mathrm{Mu}$ sic Education Research, 20(3), 277-288. doi: 10.1080/14613808.2017.1327947

Mohan, A., \& Thomas, E. (2020). Effect of background music and the cultural preference to music on adolescents' task performance. International Journal of Adolescence and Youth, 25 (1), 526-573. doi: 10.1080/02673843.2019.1689368

Moreno, R., \& Mayer, R. E. (2000). A coherence effect in multimedia learning: The case for minimizing irrelevant sounds in the design of multimedia instructional messages. Journal of Educational Psychology, 92(1), 117-125. doi: 10.1037/0022-0663.92.1.117

Mowsesian, R., \& Heyer, M. (2018). The Effect of Music as a Distraction on Test-Taking Performance. Measurement and Evaluation in Guidance, $\quad 6(2), \quad$ 104-110. doi: 10.1080/00256307.1973.12022580 
Murray, J. (2016). Impact of a Music Program on Students' Standardized Test Scores. Minneapolis: Walden University.

Neville, H., Andersson, A., Bagdade, O., Bell, T., Currin, F., Fanning., ... \& Yamada, Y. (2008). Effects of music training on brain and cognitive development in under-privileged 3- to 5-yearolds preliminary results. New York: The Dana Foundation.

Ni, C., Tsai, W., Lee, L., Kao, C., \& Chen, Y. (2012). Minimising Preoperative Anxiety with Music for Day Surgery Patients. Journal of Clinical Nursing, 21(5-6), 620-625. doi: 10.1111/j.13652702.2010.03466.x

Olsen, G. (2013). Creating the Contrast: The Influence of Silence and Background Music on Recall and Attribute Importance. Journal of Advertising, 24(4), 29-44. doi: 10.1080/00913367.1995.10673487

Oswald, C., Tremblay, S., \& Jones, D. (2000). Disruption of comprehension by meaning of irrelevant sound. Memory, 8(5), 345-350. doi: $10.1080 / 09658210050117762$

Patston, L., Hogg, S., \& Tippett, L. (2007). Attention in musicians is more bilateral than in nonmusicians. Laterality, 12(3), 262-272. doi: $10.1080 / 13576500701251981$

Pauwels, E., Volterrani, D., Mariani, G., \& Kostkiewics, M. (2014). Mozart, Music and Medicine. Medical Principles and Practice, 23(5), 403412. doi: 10.1159/000364873

Penagos-Corzo, J., y De La Fuente, R. (2012). Efectos de las características físicas y semánticas de estímulos auditivos en la atención exógena. Enseñanza e Investigación en Psicología, 17(2), 361-375. Recuperado de: https:// www.redalyc.org/pdf/292/29224159010.pdf

Perham, N., \& Currie, H. (2014). Does listening to preferred music improve reading comprehension performance? Applied Cognitive Psychology, 28(1), 279-284. doi: 10.1002/acp.2994

Perham, N., \& Vizard, J. (2011). Can preference for background music mediate the irrelevant sound effect? Applied Cognitive Psychology, 25(4), 625-631. doi: 10.1002/acp.1731

Perham, N., \& Sykora, M. (2012). Disliked music can be better for performance than liked music. Applied Cognitive Psychology, 26(1), 550-555. doi: 10.1002/acp.2826

Pool, M., Koolstra, C., \& Van Der Voort, T. (2003). Distraction Effects of Background Soap Operas on Homework Performance: An Experimental study enriched with observational data. Educa- tional Psychology, 23(4), 361-380. doi: $10.1080 / 01443410303211$

Ransdell, S., \& Gilroy, L. (2001). The effects of background music on word processed writing. Computers in Human Behavior, 17(1), 141-148. Recuperado en: https:/www.gwern.net/docs/ music-distraction/2001-ransdell.pdf

Rauscher, F., Shaw, G., Levine, L., Wright, E., Dennis, W., \& Newcomb, R. (2016). Music training causes long-term enhancement of preschool children's spatial-temporal reasoning. Neurological Research, 17(1), 2-8. doi: 10.1080/01616412.1997.11740765

Rauscher, F., \& Shaw, H. (1998). Key Components of the Mozart Effect. Perceptual and Motor Skills, 83(3 Pt 1), 835-841. doi: 10.2466/ pms.1998.86.3.835.

Rausher, F., \& Hinton, S. (2010). The Mozart Effect: Music Listening is Not Music Instruction. Educational Pschologist, 41(1), 233-238. doi: 10.1207/s15326985ep4104_3

Reynolds, J., McClelland, A., \& Furnham, A. (2014). An investigation of cognitive test performance across conditions of silence, background noise and music as a function of neuroticism. Anxiety, Stress \& Coping, 27(4), 410-421. doi: 10.1080/10615806.2013.864388

Robertson, A., \& Detmer, M. (2019). The effects of contingent lullaby music on parent-infant interaction and amount of infant crying in the first six weeks of life. Journal of Pediatric Nursing, 46(1), 33-38. doi: 10.1016/j.pedn.2019.02.025

Rose, D., Bartoli, J., \& Heaton, P. (2019). Formalinformal musical learning, sex and musicians' personalities. Personality and Individual Differences, 142(1), 207-2013. doi: 10.1016/ j.paid.2018.07.015

Rosen, L., Carrier, M., \& Cheever, N. (2013). Facebook and texting made me do it: Media-induced task-switching while studying. Computers in Human Behavior, 29(3), 948-958. doi: 10.1016/ j.chb.2012.12.001

Sala, G., \& Gobet, F. (2017). When the music's over. Does music skill transfer to children's and young adolescents' cognitive and academic skills? A meta-analysis. Educational Research Review, 20(1), 55-67. doi: 10.1016/ j.edurev.2016.11.005

Salamé, P., \& Baddeley, A. (2007). Effects of background music on phonological short-term memory. The Quarterly Journal of Experimental Psychology Section A, 41(1), 107-122. doi: $10.1080 / 14640748908402355$ 
Salmon, A. (2009). Using music to promote children's thinking and enhance their literacy development. Early Child Development and Care, 180 (7), 937-945. doi: 10.1080/03004430802550755

Schiller, I., Morsomme, D., \& Remacle, A. (2018). Voice Use Among Music Theory Teachers: A Voice Dosimetry and Self-Assessment Study. Journal of Voice, 32(5), 578-584. doi: 10.1016/ j.jvoice.2017.06.020

Schlaug, G., Norton, A., Overy, K., \& Winner, E. (2005). Effects of music training on the child's brain and cognitive development. Annals of the New York Academy of Sciences, 1660(1), 219230. doi: 10.1196/annals.1360.015

Schlaug, G. (2015). Musicians and music making as a model for the study of brain plasticity. Progress in Brain Research, 217(1), 37-55. doi: 10.1016/ bs.pbr.2014.11.020

Schmithorst, S., \& Holland, S. (2004). The effect of musical training on the neural correlates of math processing: a functional magnetic resonance imaging study in humans. Neuroscience Letters, 354(3), 193-196. doi: 10.1016/ j.neulet.2003.10.037

Seaborne, A., \& Fiorella, L. (2018). Effects of background chewing sounds on learning: The role of misophonia sensitivity. Applied Cognitive Psychology, 32(2), 4-12. doi: 10.1002/acp.3387

Shang, J., \& Gao, X. (2014). Background music matters: Why video games lead to increased aggressive behavior? Entretainment Computing, 5(2), 91-100. doi: 10.1016/j.entcom.2014.01.002

Simhon, V., Elefant, C., \& Orkibi (2018). Associations between Music and the Sensory System: Integrative Review for Child Therapy. The Arts is Psychotherapy, (In Press). doi: 10.1016/ j.aip.2018.11.005

Simpkins, S., Vest, A., \& Becnel, J. (2010). Participating in sport and music activities in adolescence: the role of activity participation and motivational beliefs during elementary school. Journal of Youth Adolescence, 39(1), 1368-1386. doi: 10.1007/s10964-009-9448-2

Snowman, J. (2010). The left and right hands of the eighteenth-century British musical prodigies, William Crotch and Samuel Wesley. Laterality: Asymmetries of Body, Brain and Cognition, 15 (2), 209-252. doi: 10.1080/13576500903201792

Swaminathan, S., Schellenberg, E., \& Venkatesan, K. (2018). Explaining the association between music training and reading in adults. Journal of Experimental Psychology: Learning, Memory, and Cognition, 44(6), 992-999. doi: 10.1037/ $\mathrm{xlm} 0000493$

Swaminathan, S., Schellenberg, G., \& Khalil, S. (2017). Revisiting the association between music lessons and intelligence: Training effects or music aptitude? Intelligence, 62(1), 119-124. doi: 10.1016/j.intell.2017.03.005

Sweller, J. (2003). Evolution of human cognitive architecture. Psychology of Learning and Motivation, 43(1), 215-266. doi: 10.1016/S0079-7421 (03)01015-6

Talamini, F., Grassi, M., Toffalini, E., Santoni, R., \& Carretti, B. (2018). Learning a second language: Can music aptitude or music training have a role? Learning and Individual Differences, 64 (1), 1-7. Doi: 10.1016/j.lindif.2018.04.003

Talero-Gutiérrez, C., \& Saade-Lemus, S. (2018). Demystifying the Mozart Effect: Facts beyond the controversy. En Gonzalez-Burgos, I. (2018). Psychobiological, Clinical and Educational Aspects of Giftedness. New York: Nova Biomedical Publishers.

Taylor, J., \& Rowe, B. (2014). The "Mozart effect" and the mathematical connection. Journal of College Reading and Learning, 42(2), 51-66. doi: 10.1080/10790195.2012.10850354

Tervaniemi, M., Szameitat, A., Kruck, S., Schröger, E., Alter, K., De Baene, W., \& Friederici, A. (2006). From air oscillations to music and speech: functional magnetic resonance imaging evidence for fine-tuned neural networks in audition. Journal of Neurosciencie, 26(34), 86478652. doi: 10.1523/jneurosci.0995-06.2006

Tierney, A., Rosen, S., \& Dick, F. (2020). Speech-inspeech perception, nonverbal selective attention, and musical training. Journal of Experimental Psychology: Learning, Memory, and Cognition, 46(5), 968-979. doi: 10.1037/ $\mathrm{x} \operatorname{lm} 0000767$

Trost, W., Ethofer, T., Zentner, M., \& Vuilleumier, P. (2012). Mapping aesthetic musical emotions in the brain. Cereb Cortex, 22(12), 2769-2783. doi: $10.1093 /$ cercor/bhr353

Trost, W., Labbé, C., \& Grandjean, D. (2017). Rhythmic entrainment as a musical affect induction mechanism. Neuropsychologia, 96(1), 91-110. doi: 10.1016/j.neuropsychologia.2017.01.004

Vidal, M., Lousada, M., \& Vigário, M. (2020). Music effects on phonological awareness development in 3-year-old children. Applied Psycholinguistics, 41(2), 299-318. doi: 10.1017/ S0142716419000535 
Wen, H., Leung, X., \& Pongtornphurt, Y. (2020). Exploring the impact of background music on customers' perceptions of ethnic restaurants: The moderating role of dining companions. Journal of Hospitality and Tourism Management, 43(1), 71-79. doi: 10.1016/j.jhtm.2020.02.007

White, E., Hutka, S., Williamsn L., \& Moreno, S. (2013). Learning, neural plasticity and sensitive periods: implications for language acquisition, music training and transfer across the lifespan. Frontiers of Neuroscience, 7(19), 90-93. doi: 10.3389/fnsys.2013.00090

Widerman, M. (2013). Study habits and music: how they affect attention and academic performance. Fairfax: George Mason University.

Wiley, J., \& Jarosz, A. (2012). How Working Memory Capacity Affects Problem Solving. Psychology of Learning and Motivation, 56(1), 185-227. doi: 10.1016/B978-0-12-394393-4.00006-6

Willis, C. (2016). Impact of Music Education on Mathematics Achievement Scores Among Middle School Students. Minneapolis: Walden University.

Wolf, A., Kopiez, R., \& Platz, F. (2018). Thinking in music: An objective measure of notationevoked sound imagery in musicians. Psychomusicology: Music, Mind, and Brain, 28(4), 209 -221. doi:10.1037/pmu0000225

Xu, S., Wang, Z., \& David, P. (2016). Media multitasking and well-being of university students. Computers in Human Behavior, 55(1), 242-250. doi: $10.1016 /$ j.chb.2015.08.040

Yilmaz, N. (2015). general music training and the place of music types used in Turkey from the ottoman period till today. Procedia, 197(1), 554 -558. doi: 10.1016/j.sbspro.2015.07.191

Zendel, N., West, G., Belleville, S., \& Peretz, I. (2019). Musical training improves the ability to understand speech-in-noise in older adults. $\mathrm{Neu}$ robiology of Aging, 81(1), 102-115. doi: 10.1016/j.neurobiolaging.2019.05.015

Zhang, H., Miller, K., Cleveland, R., \& Cortina, K. (2018). How listening to music affects reading: Evidence from eye tracking. Journal of Experimental Psychology: Learning, Memory, and Cognition, 44(11), 1778-1791. doi: 10.1037/xlm0000544 . 